\title{
Investigation of the algT operon sequence in mucoid and non-mucoid Pseudomonas aeruginosa isolates from 115 Scandinavian patients with cystic fibrosis and in 88 in vitro non-mucoid revertants
}

\begin{abstract}
Correspondence
Oana Ciofu

o.ciofu@immi.ku.dk
\end{abstract}

Received 10 July 2007

Revised 9 October 2007

Accepted 24 October 2007

\section{Oana Ciofu, ${ }^{1}$ Baoleri Lee, ${ }^{1}$ Marie Johannesson, ${ }^{3}$ Nils Olav Hermansen, ${ }^{4}$ Peter Meyer, ${ }^{5}$ Niels Høiby ${ }^{1,2}$ and the Scandinavian Cystic Fibrosis Study Consortium}

\author{
${ }^{1}$ Institute of International Health, Immunology and Microbiology, University of Copenhagen, \\ Rigshospitalet, Copenhagen, Denmark \\ ${ }^{2}$ Department of Clinical Microbiology, University Hospital, Rigshospitalet, Copenhagen, Denmark \\ 3Uppsala CF Center, Department of Women's and Children's Health, Uppsala University, \\ Uppsala University Hospital, SE-75185 Uppsala, Sweden \\ ${ }^{4}$ Department of Microbiology, Ullevaal University Hospital, Oslo, Norway \\ ${ }^{5}$ Lund CF Center, Children Hospital Lund, 22185 Lund, Sweden
}

Pseudomonas aeruginosa is the dominant pathogen causing chronic lung infections in patients with cystic fibrosis (CF). After an initial phase characterized by intermittent colonizations, a chronic infection is established upon conversion of $P$. aeruginosa from the non-mucoid to the mucoid, alginate-overproducing phenotype. During the chronic infection the isolation of both mucoid and non-mucoid isolates in CF sputum samples is very common. The purpose of the present study was to establish, by sequence analysis, the types of mutations present in the algTmucABD operon in a large number of mucoid and non-mucoid $P$. aeruginosa isolates from Scandinavian CF patients and in in vitro-derived non-mucoid revertants. Mucoid (83) and non-mucoid isolates (103) from 91 Scandinavian patients with chronic $P$. aeruginosa infection and 24 non-mucoid isolates from intermittently colonized CF patients were investigated. In addition, 88 spontaneous non-mucoid revertants obtained in vitro from nine mucoid CF isolates were also included in the study. Mutations in mucA were found in $92 \%$ of the mucoid and in up to $70 \%$ of the non-mucoid isolates from chronically infected patients, indicating that the majority of non-mucoid isolates are revertants. None of the non-mucoid isolates from intermittently colonized CF patients harboured mucA mutations. Although algT has been considered an important gene for secondary-site mutations responsible for reversion to non-mucoidy, only $30 \%$ of the mucA-mutated non-mucoid CF isolates had mutations in algT. In contrast, $83 \%$ of the in vitro-derived spontaneous non-mucoid revertants had mutations in alg $T$, showing that in the CF lung there is a selection for non-mucoid revertants with secondary-site mutations in genes other than algT. In addition, we report, to our knowledge for the first time, loss-of-function mutations in the negative regulators mucB and $m u c D$ in CF clinical isolates. In some of the CF isolates these mutations are associated with moderate alginate production. In conclusion, most non-mucoid isolates from chronically infected CF patients are revertants and the mechanism of revertance is algT-independent in the CF lung.

\section{INTRODUCTION}

Establishment of the Pseudomonas aeruginosa chronic lung infection in the cystic fibrosis (CF) lung is preceded by a period of intermittent colonization with $P$. aeruginosa strains typically resembling those found in the environment, i.e. non-mucoid and antibiotic sensitive (Doggett et al., 1966; Høiby, 1977). This stage is temporary and represents a window of opportunity for the aggressive treatment of the infection (Frederiksen et al., 1997; Høiby et al., 2005). Untreated, the infection will eventually become chronic, characterized by the appearance of 
mucoid P. aeruginosa isolates (Doggett et al., 1966; Høiby, 1977; Pedersen, 1992) and an inflammatory response dominated by polymorphonuclear leukocytes (PMNs) (Høiby et al., 1993). It has been shown that the reactive oxygen species liberated by PMNs can cause conversion of $P$. aeruginosa from non-mucoid to mucoid (Mathee et al., 1999). Besides the oxygen radicals, various other stress conditions occur in the CF lung environment, such as high osmolarity, dehydration, nutrient limitation and antibiotics, all of which may affect alginate production (Bayer et al., 1990; Berry et al., 1989; DeVault et al., 1990; Speert et al., 1990; Terry et al., 1991; Bagge et al., 2004).

Once the infecting bacteria become mucoid, the infection is very difficult to eradicate due to the biofilm mode of growth (Høiby et al., 2005). Clinically, the presence of mucoid variants is associated with poor prognosis, deterioration of the lung function and increased tissue damage (Pedersen, 1992). The main characteristic of the mucoid phenotype is the production of a thick mucopolysaccharide layer consisting of the exopolysaccharide alginate (Evans \& Linker, 1973). Great phenotypic heterogeneity has been shown among mucoid and nonmucoid CF isolates, including colony morphology, biofilm formation properties, antibiotic resistance and mediadependent alginate production (Fyfe \& Govan, 1980; Govan et al., 1983; Pugashetti et al., 1982; Drenkard \& Ausubel, 2002; Haussler, 2004; Lee et al., 2005).

The alginate biosynthesis operon (algD-algA, PA3540PA3551) is under the control of the algD promoter. A key element in algD gene regulation is the alternative sigma factor $\operatorname{Alg} \mathrm{T}$ (also termed $\mathrm{Alg} \mathrm{U}$ or $\sigma^{22}$ ), which induces the expression of algD and increases the expression of regulatory proteins that enhance $\operatorname{alg} D$ transcription (Govan \& Deretic, 1996). The algT gene belongs to an operon with four other genes, mucA-mucB-mucC-mucD.

Mutations in either mucA, $m u c B$ or $m u c D$ can lead to conversion to mucoidy (Martin et al., 1993a, b; Goldberg et al., 1993; Wood \& Ohman, 2006), suggesting that the products of these three genes have negative regulator effects on algT. While mucA mutants are highly mucoid, mucB mutants have a slightly mucoid phenotype (Martin et al., 1993a; Mathee et al., 1997), suggesting that MucA is the primary control element of AlgT activity. Mutations in $P$. aeruginosa $m u c D$ confer alginate production during growth on certain media (e.g. Pseudomonas isolation agar), suggesting that MucD can also act as a negative effector of AlgT activity (Boucher et al., 1996).

MucA acts as an anti-sigma factor that binds and sequesters $\mathrm{AlgT}$, thus affecting its ability to transcribe (Schurr et al., 1996). MucB is also a negative regulator that binds to MucA and forms a signal transduction complex that normally keeps the AlgT transcriptional activity low (Schurr et al., 1996; Mathee et al., 1997; Rowen \& Deretic, 2000). The function of MucC is generally unknown but it has been reported as being both a positive and a negative regulator (Boucher et al., 1997a). MucD is a close homologue of Escherichia coli $\mathrm{HtrA}$ (DegP), a periplasmic serine protease that is involved in the proteolysis of abnormal proteins and is required for resistance to oxidative and heat stress (Boucher et al., 1997b). It has been shown that the proteolytic motif of MucD is important for its regulation of alginate production as well as temperature resistance (Wood \& Ohman, 2006). The $m u c D$ gene also has a secondary promoter, independent of AlgT, within the algT operon (Wood \& Ohman, 2006).

Activation from a distance is a unique feature of the $\operatorname{alg} D$ promoter. Besides the control exerted by AlgT, MucA and MucB, the response regulators AlgR (Deretic et al., 1989; Nikolskaya \& Galperin, 2002), AlgB (Wozniak \& Ohman, 1991; Goldberg \& Dahnke, 1992) and the DNA-binding protein AlgZ (Baynham et al., 1999) also exert control of algD transcription.

Besides controlling alginate production, $\operatorname{Alg} \mathrm{T}$ is the $P$. aeruginosa equivalent of the extreme heat-shock sigma factor $\sigma^{\mathrm{E}}$ of E. coli (Yu et al., 1995). Congruent with the functions played by the $\sigma^{\mathrm{E}}$ in E. coli, AlgT is involved in the regulation of a number of other systems in $P$. aeruginosa, such as heat shock, osmotic protection and protection against reactive oxygen species (Martin et al., 1994; Yu et al., 1995; Firoved et al., 2002). It has also been shown that AlgT represses flagellum biosynthesis in P. aeruginosa (Tart et al., 2006). Thus, the presence of uncontrolled AlgT in mucoid isolates interferes with the normal physiology of the cell and therefore the selective advantage of mucoid forms must be critical for the ability of this organism to persistently colonize and chronically infect CF patients (Schurr et al., 1994). Besides, bacteria use a lot of energy to produce alginate and therefore it is not surprising that the mucoid phenotype is highly unstable in the absence of the selective pressure, e.g. once the strains are taken out from the CF lung (Govan et al., 1979; Ohman, 1981).

Non-mucoid isolates are often isolated from the CF sputum samples with or without simultaneous isolation of the mucoid isolates (Doggett, 1969; Høiby, 1975). These non-mucoid isolates are either wild-type $P$. aeruginosa strains or revertants from the mucoid isolates (Schurr et al., 1994). The revertants might occur by repair of the mutation in $m u c A$, or by secondary-site mutations in algT or other alginate regulatory genes (DeVries \& Ohman, 1994). Inactivation of $\operatorname{alg} T$ is the most frequent mechanism that has been demonstrated for revertants obtained in vitro (DeVries \& Ohman, 1994; Schurr et al., 1994). However, this is not the only mechanism of conversion to the nonmucoid phenotype; several alternative pathways have been suggested for the reversion to the non-mucoid state (Schurr et al., 1994). It has been shown that the nonmucoid revertants due to mutations in algT become motile through upregulation of the flagella and this was considered an adaptive mechanism allowing $P$. aeruginosa to swim towards areas of higher oxygen concentration (Wyckoff et al., 2002; Tart et al., 2006). However, the nature of the non-mucoid isolates has not previously been 
investigated on a large number of $P$. aeruginosa isolates collected from the sputum of CF patients.

In a recent study of 37 sequential isolates from $10 \mathrm{CF}$ patients attending the Hannover CF Clinic, Germany, mucA mutations were found in both mucoid and nonmucoid isolates but the occurrence of secondary-site mutations responsible for the reversion to the non-mucoid phenotype was not investigated (Bragonzi et al., 2006).

The purpose of the present study was to analyse the mutations in the algTmucABD operon, which encodes the most important regulators of alginate production. This was performed in a large collection of mucoid and non-mucoid isolates from the sputum of Scandinavian CF patients with chronic or intermittent $P$. aeruginosa infection as well as in in vitro-obtained non-mucoid revertants. The correlation between the genotype of the alginate regulatory genes and the alginate morphotypes is discussed.

\section{METHODS}

CF patients, bacterial strains and growth conditions. One hundred and sixty-six paired mucoid and non-mucoid isolates from 83 Scandinavian CF patients with chronic $P$. aeruginosa lung infection were investigated (Denmark, 38 patients; Sweden, 26 patients; and Norway, 19 patients). These patients were randomly chosen from the CF patients attending six different CF clinics (total number of CF patients/number of chronically infected with $P$. aeruginosa): Copenhagen $(240 / 112)$ and Århus (119/73) in Denmark; Uppsala (44/21) and Lund (109/27) in Sweden; and Oslo and Bergen (201/73) in Norway. Identification of the different $P$. aeruginosa clones in the six CF centres done by PFGE showed a number of different $P$. aeruginosa clones harboured by a number of patients as follows: Copenhagen, 21 different clones/twelve to two patients; Arhus, nine different clones/four to two patients; Oslo, two different clones/ten to nine patients; Uppsala, one clone/three patients; and Lund, three clones/four to two patients. Since the Arhus centre was established in 1991 and a number of CF patients transferred from Copenhagen, five clones were common, each harboured by four to two patients. The 83 pairs of mucoid and non-mucoid isolates included in this study had similar PFGE patterns and therefore were clonally related. Twenty non-mucoid isolates from eight Danish chronically infected CF patients from whom only non-mucoid colonies were routinely identified were also included in the study.

In addition, non-mucoid isolates from 24 early intermittently colonized Danish CF patients were included in this study.

The CF patients were considered chronically infected when $P$. aeruginosa was cultured in the sputum for six consecutive months or serum contained $\geqslant 2$ precipitating antibodies to $P$. aeruginosa by crossed-immunoelectrophoresis (Høiby, 1977).

Sputum samples obtained by expectoration or endolaryngeal suction were Gram-stained and examined under the microscope to confirm the origin from the lower airways, with the exception of the samples from Norway. The sputum samples of the 83 Scandinavian CF patients were plated on Blue agar plates (a modified Conrad Drigalski medium selective for Gram-negative rods, Statens Serum Institute, Copenhagen, Denmark, containing: peptone $10 \mathrm{~g}$, yeast extract $5 \mathrm{~g}$, $\mathrm{NaCl} 5 \mathrm{~g}$, agar $11 \mathrm{~g}$, detergent $0.05 \mathrm{~g}$, sodium thiosulphate $1 \mathrm{~g}$, bromthymolblue $0.1 \mathrm{~g}$, lactose $9 \mathrm{~g}$ and glucose $0.4 \mathrm{~g}$ ).

One mucoid and one non-mucoid P. aeruginosa isolate, which were simultaneously present in the sputum samples of the 83 Scandinavian patients, were collected from the Blue agar plate and considered a pair of isolates.

The sputum samples of the eight Danish CF patients who were registered in the records as presenting only non-mucoid isolates were plated on PIA (Pseudomonas isolation agar, Difco, containing peptone $20 \mathrm{~g}$, magnesium chloride $1.4 \mathrm{~g}$, potassium sulphate $10 \mathrm{~g}$, Irgasan $25 \mathrm{mg}$ and agar $13.6 \mathrm{mg}$ ), LB and Blue agar plates and incubated for at least 7 days. Colonies with different morphotypes were observed and a total of 20 colonies were isolated (one and up to four different colonies per sputum sample).

From the sputum samples of CF patients with intermittent colonization only non-mucoid isolates were cultured and one isolate from each patient was collected. All isolates were stored at $-80{ }^{\circ} \mathrm{C}$ in broth supplemented with $10 \%$ glycerol.

Spontaneous non-mucoid revertants isolated in vitro. Nine different mucoid CF isolates and PAO1 mucA22 (Mathee et al., 1999) were grown for 4-7 days in continuous culture in flow-cell biofilms as previously described (Lee et al., 2005). The bacteria recovered from biofilm reverted to the non-mucoid phenotype in a proportion of $80 \%$, and 17 different non-mucoid revertants were isolated.

The nine different mucoid CF isolates and PAO1 mucA22 were also grown in shaking aerobic conditions for 4 or 7 days and 12 different spontaneous non-mucoid revertants were isolated.

In addition, 60 separate shaking cultures, 30 in aerobic and 30 in anaerobic conditions, of one CF mucoid isolate were performed. One non-mucoid revertant from each of the 60 cultures was isolated.

In all in vitro experiments the growth medium was $\mathrm{FB}$ minimal medium $\left[1 \mathrm{mM} \mathrm{MgCl}_{2}, 0.1 \mathrm{mM} \mathrm{CaCl}_{2}\right.$, non-chelated trace elements, $2 \mathrm{~g}\left(\mathrm{NH}_{4}\right)_{2} \mathrm{SO}_{4} \mathrm{l}^{-1}, 6 \mathrm{~g} \mathrm{Na}_{2} \mathrm{HPO}_{4} \cdot 2 \mathrm{H}_{2} \mathrm{O} \mathrm{l} \mathrm{l}^{-1}, 3 \mathrm{~g} \mathrm{KH}_{2} \mathrm{PO}_{4} \mathrm{l}^{-1}, 3 \mathrm{~g}$ $\mathrm{NaCl} 1^{-1}$ ] supplemented with $0.2 \%$ Casamino acids and $0.2 \%$ glucose. Anaerobic growth was performed in the presence of $0.2 \%$ $\mathrm{KNO}_{3}$ in an anaerobic chamber $\left(85 \% \mathrm{~N}_{2}, 5 \% \mathrm{CO}_{2}, 10 \% \mathrm{H}_{2}\right.$; Don Whitley Scientific).

Investigation of the alginate morphotypes on PIA and LB media. Different alginate morphotypes were classified according to Schurr et al. (1994) on the basis of their phenotypes on two media, PIA and LB (Fig. 1). Type I was mucoid on PIA and LB; type II was mucoid on PIA but lost its mucoid appearance on LB; type III was non-mucoid on both PIA and LB; and type IV displayed very slight but detectable mucoidy on both media after an incubation period of at least 4 days (Boucher et al., 1996; Schurr et al., 1994). In order to classify the 83 pairs of mucoid and non-mucoid isolates into the different phenotypes, the isolates were subsequently grown on Blue agar plates, PIA plates and LB plates and followed for 7 days. The 20 isolates of the eight $\mathrm{CF}$ patients with chronic non-mucoid $P$. aeruginosa were recovered after prolonged growth of the sputum samples on the three different media.

Genotyping by PFGE. All isolates were genotyped by PFGE as described previously using SpeI enzyme (Ojeniyi et al., 1991; Römling \& Tümmler, 2000). After PFGE, the band patterns were visualized by ethidium bromide staining and then photographed (GelDoc imaging system, Bio-Rad). The patterns were analysed by Fingerprinting II software (Bio-Rad). The clonal relatedness of the individual pairs of mucoid and non-mucoid $P$. aeruginosa was confirmed according to Tenover et al. (1995). Isolates with PFGE patterns that differed from each other by two to three bands were considered clonally related, as this pattern is consistent with a single genetic event, a point mutation or an insertion or deletion of the DNA. Isolates with PFGE patterns that differed by more than three bands were considered to belong to different strains. 

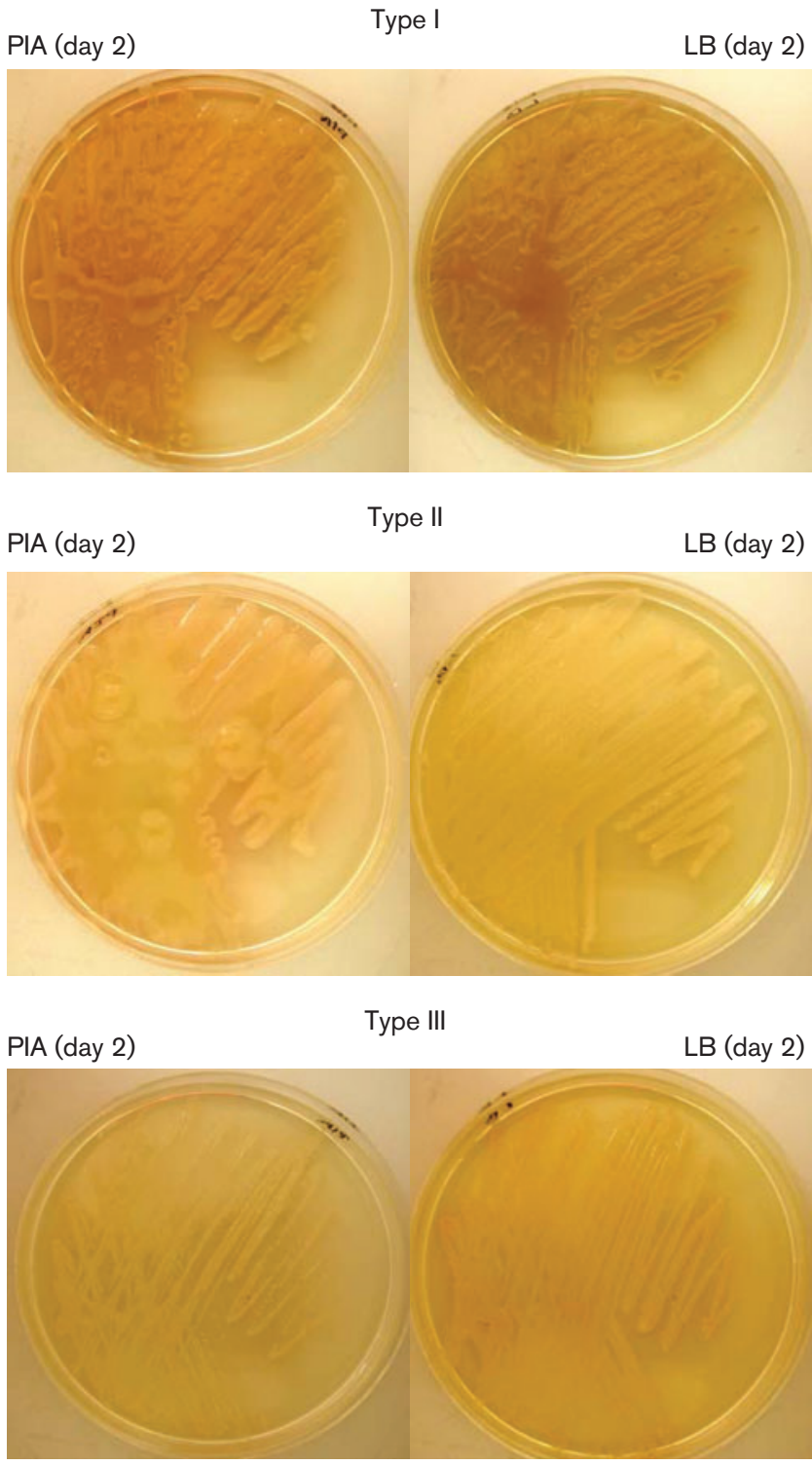

PIA (day 7)

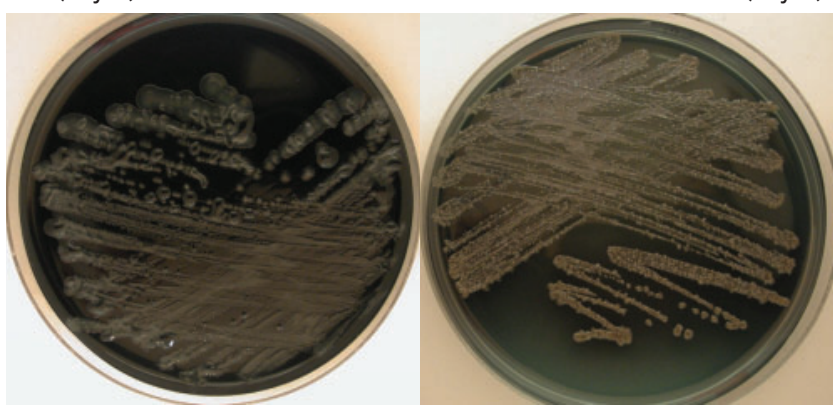

Fig. 1. Examples of the four alginate morphotypes on the basis of their phenotypes on two media, PIA (left) and LB (right), after 2 days incubation at $37{ }^{\circ} \mathrm{C}$. Type I was mucoid on both PIA and LB; type II was mucoid on PIA but lost its mucoid appearance on LB; type III was non-mucoid on both PIA and LB. After 7 days incubation at $37{ }^{\circ} \mathrm{C}$ type IV showed increased alginate production on PIA and remained non-mucoid on LB.
Sequence analysis of algTmucABD operon. DNA extraction was carried out according to the manufacturer using a commercial DNA isolation kit (Puregene, Gentra Systems).

For PCR amplification and sequencing of $\operatorname{alg} T, m u c A, m u c B$ and $m u c D$, the primers shown in Table 1 were used. PCR amplification of the entire algTmucABD operon was carried out using DYNAzyme EXT DNA polymerase (FINNZYMES).

The sequencing was done on a Macrogen automatic DNA sequencer ABI3700. The numbers of reads were between two and four for each gene of each strain. The sequence results were compared with the strain PAO1 sequence (www.pseudomonas.com) with DNASIS MAX version 2.0 (Hitachi Software Engineering), in order to determine the occurrence of sequence variants within the alg $T$ operon.

Complementation analysis. Triparental mating was used to mobilize the algT-containing recombinant plasmid pCD100 from $E$. coli JM109 to non-mucoid $P$. aeruginosa isolates showing a mucA algT mutant genotype with the conjugation helper plasmid pRK600 (Kessler et al., 1992), as previously described (Hoffmann et al., 2005). Transconjugants of $P$. aeruginosa were selected on PIA agar containing $80 \mu \mathrm{g}$ tetracycline $\mathrm{ml}^{-1}$, and complementation of algT mutations was scored by the mucoid phenotype observed on PIA plates after incubation at $37{ }^{\circ} \mathrm{C}$ to confirm that the mutations found in $\operatorname{alg} T$ were responsible for the non-mucoid phenotype.

Alginate measurement. Alginate production was measured using a borate/carbazol method (Knutson \& Jeanes, 1968) on overnight cultures in ox broth of the 83 pairs of mucoid and non-mucoid $P$. aeruginosa isolates from chronically infected CF patients. DMannuronate lactone (Sigma) was used to calibrate a standard curve, and PAO1 and PAO1 mucA22 were used as control strains. The results are presented as medians (range) and the unpaired $t$-test (Statview 4.5 software, Abacus Concepts) was used to compare the different alginate morphotype groups. Our group has previously shown that this method is specific for alginate (Pedersen et al., 1989).

Table 1. Primers used to amplify and sequence the genes in the algTmucABD operon

\begin{tabular}{|ll|}
\hline Primer & \multicolumn{1}{c|}{ Sequence $\left(\mathbf{5}^{\prime} \mathbf{- 3}^{\prime} \mathbf{)}\right.$} \\
\hline algT-sek1 & CCTGAGCCCGATGCAATCCATTTTCG \\
algT-sek2 & GGTATGCCTTGATGAAGGCTTCCTGC \\
algT-sek3 & GCTGTATCGGATCGCCATCAACACC \\
algT-sek4 & GGACAGAGTTTCCTGCAGGGCTTCAC \\
mucA-1 & CTCTGCAGCCTTTGTTGCGAGAAG \\
mucA-1 rev & CTGCCAAGCAAAAGCAACAGGGAGG \\
mucA-2 & GTGCGTCTGTACAACCAGAACGACG \\
mucA-2rev & GTCGTTCTGGTTGTACAGACGCACG \\
mucB-1 & ATCCGCCGTCAGTGGTACAG \\
mucB-1 rev & CGAGCAGGACGAGCAGGTAC \\
mucB-int1 & CAGTGGTCCTTGCGGTGACT \\
mucB-int2 & TTCAGCAGCAGCGACTTCAA \\
mucD-1 & GTCCGATTCGGCCTGAGTCT \\
mucD-1 rev & ACGCAGGTAACGGATTGACG \\
mucD-int1 & GATCAACCCGGGTAACTCCG \\
mucD-int2 & AGATCTGCGAGTTGATGCCG \\
mucD-int3 & CATCCTCACCAACAATCACGTCGTGG \\
mucD-int4 & GCTCGCTACGGTCGGACAGG \\
mucD-int5 & CAGCGCAAGTCCCTGAGCATGG \\
mucD-int6 & GCTTCATGTTGCCCACCAGGTGC \\
& \\
\hline
\end{tabular}




\section{RESULTS}

\section{Clonal distribution of the mucoid and non-mucoid isolates}

Among the 38 Danish patients with chronic P. aeruginosa infection, two dominant clones were found: Dk1 (16 patients) and Dk2 (9 patients). CF patients from both the Copenhagen and the Århus CF centres harboured these clones. The presence of these two dominant clones was published previously by our group (Ojeniyi et al., 1993; Jelsbak et al., 2007). Among the 26 Swedish patients, three different clones were found among patients attending the Lund CF centre: L1 (three patients), L2 and L3 (each infecting two patients). One patient from Lund and one patient from Arhus harboured the same clone. Among the 19 Norwegian patients, one dominant clone was found, clone $\mathrm{N}$ (13 patients). The presence of this dominant clone among Norwegian CF patients was published previously (Fluge et al., 2001). Unique clones among P. aeruginosa isolates from Denmark (Ud), Sweden (Us) and Norway (Un) were found in the rest of the patients.

Among the $24 \mathrm{CF}$ patients with intermittent colonization, three clones, each harboured by two patients, were found, while the rest of the patients harboured unique clones.

The genotyping analysis showed that with the exception of one patient from Sweden and one from Denmark who shared the same clone, no cross-infection occurred among the Danish, Swedish and Norwegian CF patients attending the six different clinics in Scandinavia. Patients who moved to a newly established clinic in Denmark harboured some of the dominant clones from the original centre.

\section{Distribution of the different alginate morphotypes}

Four alginate morphotypes could be distinguished, in agreement with Schurr et al. (1994): see Fig. 1.

The alginate phenotype of the mucoid and non-mucoid isolates from the 83 chronically infected CF patients was initially determined on Blue agar plates. Subsequent culture on PIA and LB plates showed that $87 \%$ of the non-mucoid and $89 \%$ of the mucoid maintained their phenotype on PIA and LB. This means that $87 \%$ of the non-mucoid isolates showed a type III alginate production and $89 \%$ of the mucoid isolates a type I alginate production.

However, six non-mucoid and eight mucoid isolates showed a phenotype of $\mathrm{Alg}^{+}$on PIA and $\mathrm{Alg}^{-}$on $\mathrm{LB}$, corresponding to a type II alginate morphotype, and five non-mucoid isolates showed slightly increased alginate production on PIA after 7 days incubation, corresponding to type IV alginate production.

From the eight CF patients with chronic infection with non-mucoid $P$. aeruginosa, five isolates showed type IV alginate production, starting to produce increased amounts of alginate after prolonged incubation, while the rest of the
15 non-mucoid isolates maintained their phenotype and showed type III alginate production.

When the phenotypic characterization of alginate production (types I, II, III and IV) as judged by the colony morphology on PIA and LB plates was compared to the level of alginate production measured on overnight culture in ox broth, a good correlation was found. The median amount of alginate measured in isolates that showed a type I, II, III and IV morphotype was 180.69 (16.8-757.9), 79.6 (1.07-449), $0(0-27.9)$ and $2(0-496) \mathrm{mg} \mathrm{l}^{-1}$, respectively. The differences between the different groups were statistically significant. This shows that colony morphology on PIA plates correlates with the amount of alginate produced by the isolates in a rich medium such as ox broth.

\section{Mutations in mucA, mucB and mucD in non- mucoid and mucoid $\boldsymbol{P}$. aeruginosa isolates from chronically infected patients}

The DNA sequence analysis of mucA carried out on nonmucoid (103) and mucoid (83) P. aeruginosa isolates from chronically infected Scandinavian CF patients showed that $71(69 \%)$ of the non-mucoid isolates and $77(93 \%)$ of the mucoid isolates had mutations in $m u c A$. No mutations in mucA were found in the non-mucoid isolates from 24 intermittently colonized patients. The types of mucA mutations in Scandinavian mucoid and non-mucoid isolates are presented in Table 2.

The most common mutation was a $\mathrm{C} / \mathrm{T}$ transition at position 349 or $352 \mathrm{bp}$ of the mucA gene ( $m u c A$ consists of $585 \mathrm{bp}$ ), which resulted in a stop codon, followed by the mucA22 mutation, a deletion of a $\mathrm{G}$ within the homopolymeric stretch of five $G$ residues located between positions 429 and 433 of the mucA coding region.

An association between the dominant clones and the types of mucA mutation was observed: clone Dk1 and T349C, clone Dk2 and mucA22, and clone N and T352C. These associations provide an explanation for the high frequency with which these types of mucA mutations were found among the Scandinavian CF isolates. However, the same types of mutations were also found in unrelated clones from different geographical locations in Scandinavia, suggesting that these loci represent hot-spot regions for mutation in mucA. In addition, the mucA22 mutation was reported previously in several mucoid isolates from the USA, Europe and Australia (Boucher et al., 1997b; Bragonzi et al., 2006; Anthony et al., 2002). Isolates belonging to the Dk1, Dk2 and $\mathrm{N}$ clones showed several other types of mutations.

The sequence alterations found in $m u c B$ and $m u c D$ in mucoid and non-mucoid isolates of the chronically infected CF patients are presented in Table 3. For mucB we identified several insertions and deletions, leading to frameshifts and premature stop codons. We also identified a $\mathrm{T}$ deletion at position 41 of $m u c D$ leading to a premature stop codon. These types of gene alterations probably result 
Table 2. Types of mutations and the respective changes determined in the mucA sequence of mucoid (M) and non-mucoid (NM) CF P. aeruginosa isolates

The number of isolates with the respective mutations and the clonal distribution of the isolates are also shown.

\begin{tabular}{|c|c|c|c|c|}
\hline Mutation in $m u c A$ & Sequence change & M (83) & NM (103) & Clones \\
\hline $\mathrm{C} 137 \mathrm{~T}$ & $\mathrm{Ala} / \mathrm{Val}$ & & 5 & $\mathrm{Ud}$ \\
\hline$\Delta \mathrm{CC} 160$ & Frameshift, stop codon 474 & 1 & & $\mathrm{Dk} 1$ \\
\hline$\Delta \mathrm{TA} 162$ & Frameshift, stop codon 474 & & 1 & $\mathrm{~N}$ \\
\hline Insertion $\mathrm{C}$ at 222 & Frameshift, stop codon 478 & 1 & & Ud \\
\hline$\Delta 14 \mathrm{bp}$ at 223 & Frameshift, stop codon 462 & 1 & 1 & Ud \\
\hline Insertion 5 bp at 253 & Frameshift, stop codon 289 & 2 & 2 & L3 \\
\hline$\Delta 5 \mathrm{bp}$ at 266 & Frameshift, stop codon 471 & 1 & & Ud \\
\hline$\Delta 14 \mathrm{bp}$ at 297 & Frameshift, stop codon 567 & 1 & 1 & Dk2 \\
\hline Insertion $4 \mathrm{bp}$ at 300 & Frameshift, stop codon 481 & 1 & 1 & $\mathrm{Ud}$ \\
\hline$\Delta 105$ bp at 307 & Frameshift, stop codon 478 & 3 & 4 & $\mathrm{~N}$ \\
\hline Insertion $\mathrm{T}$ at 326 & Frameshift, stop codon 478 & 1 & 1 & Dk1 \\
\hline$\Delta 42$ bp at 339 & Frameshift, stop codon 541 & & 2 & $\mathrm{~N}$ \\
\hline $\mathrm{C} 340 \mathrm{~T}$ & Stop codon & 1 & 3 & Us \\
\hline T349C & Stop codon & 15 & 16 & Dk1, Un, Ud \\
\hline $\mathrm{T} 352 \mathrm{C}$ & Stop codon & 14 & 8 & $\mathrm{~N}, \mathrm{~L} 2, \mathrm{Ud}, \mathrm{Us}$ \\
\hline$\Delta \mathrm{G} 357$ & Frameshift, stop codon 385 & 1 & 1 & Us \\
\hline$\Delta \mathrm{G} 358$ & Frameshift, stop codon 478 & 1 & & Us \\
\hline$\Delta \mathrm{C} 360$ & Frameshift, stop codon 385 & 1 & 1 & Un \\
\hline$\Delta \mathrm{C} 365$ & Frameshift, stop codon 385 & 2 & 1 & Ud, Un \\
\hline T367C & Stop codon & 1 & 1 & Us \\
\hline$\Delta 19$ bp at 368 & Frameshift, stop codon 420 & 1 & 1 & Ud \\
\hline Insertion $\mathrm{C}$ at 368 & Frameshift, stop codon 478 & 1 & & $\mathrm{Ud}$ \\
\hline $\mathrm{C} 382 \mathrm{~T}$ & Stop codon & 1 & & $\mathrm{~L} 2$ \\
\hline G421T & Stop codon & 1 & & $\mathrm{~L} 1$ \\
\hline $\mathrm{C} 424 \mathrm{~T}$ & Stop codon & 2 & 1 & Us \\
\hline$\Delta \mathrm{G} 430$ & Frameshift, stop codon 439 & 21 & 18 & Dk2, Us, Un, Ud \\
\hline Insertion CG at 431 & Frameshift, stop codon 441 & 1 & & Us \\
\hline Insertion GCG at 431 & Frameshift & & 1 & Us \\
\hline$\Delta \mathrm{G}$ at 432 & Frameshift, stop codon 439 & 1 & 1 & Un \\
\hline
\end{tabular}

in loss of function of their gene products and have apparently not been reported before. Some isolates had several point mutations leading to multiple amino acid changes in MucB or MucD. However, in a large number of the CF isolates simultaneous mutations in $m u c A$ and $m u c B$ or $m u c D$ were identified (results not shown). Therefore, the alginate regulator roles played by $m u c B$ and $m u c D$ are difficult to assess from our data. We identified five isolates with sequence alterations in $m u c B$ or $m u c D$ and wild-type mucA. One isolate showed a mucoid phenotype only on PIA (type II) and four isolates showed a mucoid phenotype after prolonged incubation (type IV) (Table 3 ). The isolates with type II alginate production had a $4 \mathrm{bp}$ insertion at position 808 in $m u c B$, resulting in a frameshift and a premature stop codon at position 855 ( $m u c B$ consists of $951 \mathrm{bp}$ ). Two of the isolates with type IV alginate production harboured a $\Delta 162 \mathrm{bp}$ at 235 and an $8 \mathrm{bp}$ insertion at 466 , resulting in premature stop codons in $m u c B$. The other two isolates with type IV alginate production showed a $\Delta \mathrm{T} 41$, resulting in a stop codon in $m u c D$ ( $m u c D$ consists of $1425 \mathrm{bp}$ ), and several point mutations in $m u c D$ (A409G, C673G, G1321A), resulting in amino acid changes (Table 3). These findings suggest that these sequence alterations might result in loss of function of the negative regulators $\mathrm{MucB}$ or $\mathrm{MucD}$, leading to increased transcription of the alginate operon when growing on PIA plates or after prolonged incubation.

Interestingly, the more common single-point mutations C338T and A631G found in $m u c B$ or A409G, C673G and G1321A in mucD that led to amino acid changes in the respective encoded proteins were reported previously in German isolates (Bragonzi et al., 2006) and might suggest that these are hot-spot regions for mutations in $m u c B$ and mucD.

\section{Mutations in the algT gene in non-mucoid $P$. aeruginosa isolates}

Of the 71 non-mucoid isolates with a mucA mutant genotype, only 37 (52\%) had sequence alterations in algT. Moreover, some algT mutations, such as $\mathrm{A} 55 \mathrm{G}$ resulting in a change of Lys to Glu (13 non-mucoid isolates), C115T resulting in a change of Arg to $\operatorname{Trp}$ (one non-mucoid 
Table 3. Types of mutations and the changes determined in the sequence of mucB and mucD in mucoid (M) and nonmucoid (NM) isolates

The number of isolates with the respective mutation is shown. Isolates with reduced alginate production (such as type II or IV) are indicated. Some isolates had several mutations in $m u c B$ and $m u c D$; these are shown by superscript numbers $\left({ }^{1-6}\right)$ for each mutation type.

\begin{tabular}{|c|c|c|c|}
\hline Mutation & Sequence change & $\begin{array}{c}\text { M } \\
(\mathbf{8 3})\end{array}$ & $\begin{array}{l}\text { NM } \\
(103)\end{array}$ \\
\hline \multicolumn{4}{|l|}{ Mutations in $m u c B$} \\
\hline A77G & Asp/Gly & 2 & 2 \\
\hline $\mathrm{C} 157 \mathrm{~T}$ & Pro/Ser & 1 & 1 \\
\hline $\mathrm{G} 179 \mathrm{~T}^{1}$ & Arg/Leu & & 1 \\
\hline C208T & Arg/Cys & & 1 \\
\hline Insertion GC at 235 & Frameshift, stop codon 418 & 1 & \\
\hline$\Delta 162 \mathrm{bp}$ at 235 & Frameshift, stop codon 787 & & $4(\mathrm{IV})$ \\
\hline $\mathrm{T} 245 \mathrm{C}^{2}$ & Val/Ala & & 1 \\
\hline $\mathrm{G}_{3} 31 \mathrm{~T}^{3}$ & Asp/Tyr & & 1 \\
\hline $\mathrm{C} 338 \mathrm{~T}^{\star 4}$ & Ser/Phe & 6 & 6 \\
\hline A356G & Tyr/Cys & 1 & 1 \\
\hline $\mathrm{T} 420 \mathrm{C}^{\star 5}$ & Glu/Asp & 1 & \\
\hline Insertion 8 bp at 466 & Frameshift & & $1(\mathrm{IV})$ \\
\hline $\mathrm{G} 468 \mathrm{~A}^{5}$ & Glu/Asp & & \\
\hline Insertion $\mathrm{A}$ at 545 & Frameshift, stop codon 853 & & 1 \\
\hline$\Delta \mathrm{GGC}$ at $550^{2}$ & Frameshift, stop codon 945 & & \\
\hline Insertion $\mathrm{A}$ at $570^{3}$ & Frameshift & & \\
\hline Insertion $\mathrm{T}$ at $576^{3}$ & Frameshift & & \\
\hline Insertion $\mathrm{A}$ at $594^{3}$ & Frameshift & & \\
\hline Insertion $44 \mathrm{bp}$ at 610 & Frameshift, stop codon 717 & & 1 \\
\hline G611A ${ }^{6}$ & Ala/Gly & 3 & 3 \\
\hline $\mathrm{A} 631 \mathrm{G}^{\star 5,6}$ & Thr/Ala & 3 & 3 \\
\hline Insertion 4 bp at $808^{4}$ & Frameshift, stop codon 855 & & (II) \\
\hline $\mathrm{A} 847 \mathrm{G}^{1}$ & Thr/Ala & & \\
\hline$\Delta \mathrm{T} 858$ & Frameshift, stop codon 867 & 1 & 1 \\
\hline Insertion $\mathrm{A}$ at 906 & Frameshift & & $1(\mathrm{IV})$ \\
\hline \multicolumn{4}{|l|}{ Mutations in $m u c D$} \\
\hline$\Delta \mathrm{T} 41$ & Stop codon & & $1(\mathrm{IV})$ \\
\hline C47T & Ala/Val & & 1 \\
\hline A163G & Met/Val & 1 & 1 \\
\hline A245G & Gln/Arg & & 1 \\
\hline $\mathrm{T} 274 \mathrm{C}^{1}$ & Cys/Arg & 1 & 1 \\
\hline G361A ${ }^{2}$ & Glu/Lys & & 1 \\
\hline $\mathrm{A} 409 \mathrm{G}^{\star 1,2,3,4}$ & Ile/Val & 42 & 39 \\
\hline $\mathrm{C} 467 \mathrm{~T}^{3}$ & Pro/Leu & & 1 \\
\hline T515G & Val/Gly & & 1 \\
\hline $\mathrm{C} 673 \mathrm{G}^{* 4}$ & Gln/Glu & 1 & 1 \\
\hline $\mathrm{A} 1004 \mathrm{~T}$ & $\mathrm{Val} / \mathrm{Ala}$ & & 1 \\
\hline A1040T & Asp/Val & 2 & 2 \\
\hline $\mathrm{G} 1321 \mathrm{~A}^{*^{4}}$ & Val/Ile & 1 & (IV) \\
\hline
\end{tabular}

${ }^{\star}$ Mutations previously reported by Bragonzi et al. (2006).

isolate), C389T resulting in a change from Ala to Val (one non-mucoid isolate) and C430T resulting in a change of Arg to Cys (one non-mucoid isolate), were also found in the algT sequence of the paired mucoid isolates, suggesting that these mutations do not affect the functionality of AlgT.
Thus, $\operatorname{alg} T$ mutations present only in isolates with a nonmucoid phenotype were found in 21 isolates representing $30 \%$ of the mucA mutant non-mucoid revertants (Table 4 ). With the exception of C58T (one isolate), C496T (one isolate), C499T (seven isolates) and a $\mathrm{T}$ deletion at position 97 (one isolate), all the other mutations could be complemented by pCD100, encoding the wild-type algT (Table 4). Although we could not confirm by complementation the effect of the $\mathrm{T}$ deletion at position 97, this mutation very probably causes a loss of function of the gene product, as it leads to a frameshift and a stop codon at position $106 \mathrm{bp}$ in $\operatorname{alg} T$ ( $\operatorname{alg} T$ consists of $582 \mathrm{bp}$ ). Even though we did not succeed in the complementation of all point mutations, we cannot exclude that the functionality of the $\operatorname{alg} T$ gene product is affected by these point mutations (Table 4). In contrast to the low percentage of CF non-mucoid revertants that had changes in $\operatorname{alg} T$, up to $83 \%$ (73 out of 88 ) of the spontaneous non-mucoid revertants obtained in vitro showed mutations in $\operatorname{alg} T$ (Table 5).

Fifteen of the 88 in vitro revertants had a wild-type algT allele and, interestingly, 14 of them were recovered after anaerobic or biofilm growth. This might suggest that under stress conditions such as low oxygen tension, a selection of isolates with impaired AlgT function might occur.

The types of $\operatorname{alg} T$ mutations found in the 21 clinical nonmucoid revertants were different from the types of mutations found in the 88 in vitro-obtained isolates. However, a 9 bp insertion found in 3 out of $21 \mathrm{CF}$ isolates was frequently observed in the in vitro isolates, especially

Table 4. Types of mutations and the determined changes in the sequence of $\operatorname{alg} T$ in non-mucoid clinical isolates with a mucA mutant genotype

The number of isolates showing the respective mutation is shown. Complementation by wild-type $\operatorname{alg} T$ is also indicated.

\begin{tabular}{|lllc|}
\hline $\begin{array}{l}\text { Mutation in } \\
\text { algT in clinical } \\
\text { isolates }\end{array}$ & Complementation & \multicolumn{1}{c|}{$\begin{array}{c}\text { Sequence } \\
\text { changes }\end{array}$} & $\begin{array}{c}\text { No. of } \\
\text { isolates } \\
(\mathbf{2 1})\end{array}$ \\
\hline C58T & & $\begin{array}{l}\text { Arg/Trp } \\
\text { Frameshift, stop }\end{array}$ & 1 \\
codon & & $\begin{array}{l}\text { Frameshift, stop } \\
\text { codon 589 }\end{array}$ & 3 \\
Insertion 9 bp & pCD100 & Stop codon & 2 \\
at 138 & pCD100 & Gln/Arg & 1 \\
C142T & pCD100 & Cys/Phe & 1 \\
A143G & pCD100 & Tyr/Phe & 1 \\
G164T & pCD100 & Leu/Pro & 1 \\
A212T & pCD100 & Gly/Asp & 1 \\
T224C & pCD100 & Gly/Cys & 1 \\
G344A & & Pro/Ser & 7 \\
G496T & & Frameshift, stop & 1 \\
C499T & pCD100 & codon 536 & \\
Insertion T at & & & \\
525 & & & \\
\hline
\end{tabular}


after aerobic growth. Interestingly, a mutation at codon 29 leading to a change of Tyr to Cys previously found by DeVries \& Ohman (1994) in non-mucoid revertants was also present among the in vitro revertants obtained in this study.

\section{DISCUSSION}

Sequence analysis of the $\operatorname{alg} T$ operon in a large number of mucoid and non-mucoid $P$. aeruginosa isolates from Scandinavian CF patients showed that the majority of the non-mucoid isolates are revertants from the mucoid phenotype as they have mutations in $m u c A$. Although $\operatorname{alg} T$ has been considered one of the main genes for secondary-site mutations resulting in reversion to nonmucoidy, inactivation of algT was found in only $30 \%$ of the $\mathrm{CF}$ revertants. However, a much higher percentage $(86 \%)$ of the in vitro spontaneous revertants showed inactivation of algT. Previous studies showed that 9 out of 28 (DeVries \& Ohman, 1994) and two out of three (Schurr et al., 1994) spontaneous revertants had mutations in $\operatorname{alg} T$.

These data show that although algT mutants can grow and survive in laboratory conditions, they are less proficient in the CF lung environment due to the role played by AlgT in adaptation to stress. The large variety of the $\operatorname{alg} T$ mutations found in the present and previous studies might suggest that $\operatorname{alg} T$ contains no hot-spot regions for mutations as is the case for its negative regulator, mucA.

The role played by AlgT in stress adaptations has been established by several studies.

Transcriptomic analysis revealed differential AlgT-dependent expression of the osmC and osmE genes, which potentially play a role in stress defence (Palma et al., 2004). Therefore, $\operatorname{alg} T$ mutants would be less proficient in adapting to osmotic stress. It has also been shown that algT mutants of $P$. aeruginosa showed increased susceptibility to reactive oxygen intermediates (Martin et al., 1994; Yu et al., 1996), indicating that even though algT mutants occur frequently in vitro (DeVries \& Ohman, 1994; Schurr et al., 1994), they are probably destroyed by the host response in vivo. Finally, in a recent paper (Wood et al., 2006) algT was identified together with $\operatorname{alg} B$ and $\operatorname{alg} R$ as an important regulator of the alginate induction by environmental factors such as cell-wall-inhibitory antibiotics. If algT activation is involved in the response to cell-wall stress, then an $\operatorname{alg} T$ mutant could be more susceptible to cell-wall antibiotics and therefore easily destroyed in the lung by the antibiotic treatment of the chronic lung infection. If this is true, we can not exclude a selection bias towards nonmucoid revertants with wild-type algT, which might be more resistant to antibiotics. Another possible explanation could be that mutations that arise during the strong

Table 5. Types of mutations and the determined sequence changes in alg $T$ found in non-mucoid revertants obtained in vitro

The conditions under which these revertants occurred (biofilm, aerobic and anaerobic growth) are shown. The numbers of isolates with the respective mutations are indicated.

\begin{tabular}{|c|c|c|c|}
\hline Mutation in $\operatorname{alg} T$ & Sequence alteration & Growth condition & No. of isolates (88) \\
\hline$\Delta 16$ bp at 21 & Frameshift, stop codon 64 & Biofilm & 1 \\
\hline $\mathrm{C} 37 \mathrm{~T}$ & Trp/Arg & Biofilm 1; anaerobic 11 & 12 \\
\hline Insertion $11 \mathrm{bp}$ at 50 & Frameshift, stop codon 90 & Anaerobic & 1 \\
\hline A86G & Tyr/Cys & Biofilm & 1 \\
\hline Insertion $\mathrm{T}$ at 97 & Frameshift, stop codon 205 & Anaerobic & 1 \\
\hline $\mathrm{C} 122 \mathrm{~T}$ & Ala/Val & Biofilm & 1 \\
\hline Insertion 9 bp at 138 & Frameshift, stop codon 589 & Aerobic 22; biofilm 4 & 26 \\
\hline G145A & Asp/Asn & Aerobic & 1 \\
\hline Insertion 9 bp at 147 & Frameshift, no stop codon & Aerobic 9; anaerobic 3 & 12 \\
\hline$\Delta \mathrm{T} 167$ & Frameshift, stop codon 294 & Aerobic & 1 \\
\hline $\mathrm{C} 182 \mathrm{~T}$ & Ala/Val & Aerobic & 1 \\
\hline Insertion $\mathrm{C}$ at 192 & Frameshift, stop codon 204 & Aerobic & 1 \\
\hline$\Delta \mathrm{T} 204$ & Frameshift, stop codon 295 & Aerobic & 1 \\
\hline$\Delta \mathrm{T} 214$ & Frameshift, stop codon 294 & Biofilm & 1 \\
\hline G230T & Arg/Leu & Biofilm & 1 \\
\hline Insertion 7 bp at 342 & Frameshift, stop codon 385 & Aerobic & 1 \\
\hline $\mathrm{C} 361 \mathrm{~T}$ & Arg/Trp & Anaerobic & 1 \\
\hline $\mathrm{C} 412 \mathrm{~T}$ & Stop codon & Biofilm & 1 \\
\hline$\Delta \mathrm{T} 476$ & Frameshift, stop codon 487 & Aerobic & 1 \\
\hline$\Delta \mathrm{G} 507$ & Frameshift, no stop codon & Aerobic & 1 \\
\hline A508G & Thr/Ala & Biofilm & 1 \\
\hline$\Delta \mathrm{G} 511$ & Frameshift, no stop codon & Anaerobic & 5 \\
\hline
\end{tabular}


selection pressure in the CF lung are different from the mutations that arise during non-selective growth in the laboratory. Interestingly, in vitro spontaneous non-mucoid isolates with wild-type alg $T$ were recovered after anaerobic or biofilm growth conditions, suggesting that the nonmucoid CF isolates might occur in the anaerobic conditions encountered in the mucus plugs present in the conductive airways of the CF lungs.

Although the existence of alternative pathways that cause revertance to the non-mucoid phenotype has been postulated, the site of these mutations is not yet known. However, the investigations of the alginate morphotypes on LB and PIA agar might suggest that mutations in the regulatory genes $\lg B$ and $\operatorname{llg} R$ could be candidates for the secondary mutations responsible for in vivo revertance. PIA medium contains Irgasan, the trade name for triclosan, which has been shown to be a strong alginate inducer by a mechanism dependent on algT, algB and $\operatorname{algR}$ (Wood et al., 2006). Thus, lack of inducibility of alginate production on PIA agar (type III alginate production) should characterize mutants in one of the three genes. Our study shows that the majority of the CF non-mucoid revertants showing a type III morphotype had no mutations in algT and therefore mutations in $\operatorname{alg} B$ and $\operatorname{alg} R$ could explain this morphotype.

The mucA mutations identified in non-mucoid CF isolates were also found in the paired mucoid isolates. In agreement with previous studies (Boucher et al., 1997b; Yoon et al., 2006; Bragonzi et al., 2006), mutations in mucA were found to be the main mechanism of conversion to mucoidy. However, we were also able to identify loss-offunction mutations in $m u c B$ or $m u c D$ in some non-mucoid isolates with increased alginate production on PIA plates (type II) or after prolonged incubation (type IV). As these non-mucoid isolates with attenuated alginate production in vitro have mutations in the alginate regulatory genes, they might have the potential for increased alginate production in vivo in the $\mathrm{CF}$ lung. In some $\mathrm{CF}$ patients with chronic $P$. aeruginosa infections no mucoid isolates are cultured. We suggest that these $P$. aeruginosa isolates have $m u c B$ or $m u c D$ mutations and might be misinterpreted as non-mucoid in the clinical microbiological laboratory.

Mucoid isolates with a wild-type mucA allele were found in $7 \%$ of the isolates, showing, in agreement with previous studies (Martin et al., 1993b), that other loci are involved in the conversion to mucoidy. However, this seems to be a rare event among Scandinavian CF isolates compared to mucoid isolates from CF clinics in the USA and Canada, where, according to Yoon et al. (2006), approximately $13 \%$ of the mucoid isolates had a wild-type mucA.

As expected, $100 \%$ of the non-mucoid strains isolated from intermittently colonized patients showed wild-type $m u c A$, confirming that the initial colonization of the CF patients occurs with environmental wild-type isolates that convert to alginate overproducers under the selection pressure in the CF lung. The demographic data showed that the intermittently colonized patients were much younger than the chronically colonized patients. These intermittently colonized patients are in the window of opportunity for the treatment of the infection before mutations in $m u c A$, responsible for conversion to mucoidy, occur.

Non-mucoid isolates from chronically colonized CF patients with wild-type mucA allele were found in $30 \%$ of the isolates, suggesting that these isolates might be the original wild-type isolates that infected the patient. However, some of these isolates showed algT mutations (data not shown), raising the possibility that acquisition of a wild-type mucA allele had occurred by horizontal gene transfer.

In conclusion, most of the non-mucoid $\mathrm{CF}$ isolates are revertants from mucoid isolates and the mechanism of revertance is algT-independent; this is in contrast to the in vitro-obtained isolates, which frequently show mutations in algT. Investigations are continuing in our laboratory to find out which genes might be responsible for the conversion to non-mucoidy in vivo.

\section{ACKNOWLEDGEMENTS}

We wish to thank Ulla Johansen, Tina Wassermann and Jette Teglhus Møller for excellent technical assistance. We acknowledge Dr Tacjana Pressler from the CF Center in Copenhagen, Dr Gjermund Fluge from the CF Center in Bergen and Dr Bengt Wretlind from the CF Center in Huddinge, Stockholm, for providing patient information. We also wish to thank Thomas Bjarnsholt for critically reading the manuscript.

\section{REFERENCES}

Anthony, M., Rose, B., Pegler, M. B., Elkins, M., Service, H., Thamotharampillai, K., Watson, J., Robinson, M., Bye, P. \& other authors (2002). Genetic analysis of Pseudomonas aeruginosa isolates from the sputa of Australian adult cystic fibrosis patients. J Clin Microbiol 40, 2772-2778.

Bagge, N., Schuster, M., Hentzer, M., Ciofu, O., Givskov, M., Greenberg, E. P. \& Høiby, N. (2004). Pseudomonas aeruginosa biofilms exposed to imipenem exhibit changes in global gene expression and beta-lactamase and alginate production. Antimicrob Agents Chemother 48, 1175-1187.

Bayer, A. S., Eftekhar, F., Tu, J., Nast, C. C. \& Speert, D. P. (1990). Oxygen-dependent up-regulation of mucoid exopolysaccharide (alginate) production in Pseudomonas aeruginosa. Infect Immun 58, 1344-1349.

Baynham, P. J., Brown, A. L., Hall, L. L. \& Wozniak, D. J. (1999). Pseudomonas aeruginosa AlgZ, a ribbon-helix-helix DNA-binding protein, is essential for alginate synthesis and $\operatorname{alg} D$ transcriptional activation. Mol Microbiol 33, 1069-1080.

Berry, A., DeVault, J. D. \& Chakrabarty, A. M. (1989). High osmolarity is a signal for enhanced $a \lg D$ transcription in mucoid and nonmucoid Pseudomonas aeruginosa strains. J Bacteriol 171, 2312-2317.

Boucher, J. C., Martinez-Salazar, J., Schurr, M. J., Mudd, M. H., Yu, H. \& Deretic, V. (1996). Two distinct loci affecting conversion to mucoidy in Pseudomonas aeruginosa in cystic fibrosis encode homologs of the serine protease HtrA. J Bacteriol 178, 511-523. 
Boucher, J. C., Schurr, M. J., Yu, H., Rowen, D. W. \& Deretic, V. (1997a). Pseudomonas aeruginosa in cystic fibrosis: role of mucC in the regulation of alginate production and stress sensitivity. Microbiology 143, 3473-3480.

Boucher, J. C., Yu, H., Mudd, M. H. \& Deretic, V. (1997b). Mucoid Pseudomonas aeruginosa in cystic fibrosis: characterization of $m u c$ mutations in clinical isolates and analysis of clearance in a mouse model of respiratory infection. Infect Immun 65, 3838-3846.

Bragonzi, A., WiehImann, L., Klockgether, J., Cramer, N., Worlitzsch, D., Doring, G. \& Tummler, B. (2006). Sequence diversity of the $m u c A B D$ locus in Pseudomonas aeruginosa isolates from patients with cystic fibrosis. Microbiology 152, 3261-3269.

Deretic, V., Dikshit, R., Konyecsni, W. M., Chakrabarty, A. M. \& Misra, T. K. (1989). The algR gene, which regulates mucoidy in Pseudomonas aeruginosa, belongs to a class of environmentally responsive genes. J Bacteriol 171, 1278-1283.

DeVault, J. D., Kimbara, K. \& Chakrabarty, A. M. (1990). Pulmonary dehydration and infection in cystic fibrosis: evidence that ethanol activates alginate gene expression and induction of mucoidy in Pseudomonas aeruginosa. Mol Microbiol 4, 737-745.

DeVries, C. A. \& Ohman, D. E. (1994). Mucoid-to-nonmucoid conversion in alginate-producing Pseudomonas aeruginosa often results from spontaneous mutations in alg $T$, encoding a putative alternate sigma factor, and shows evidence for autoregulation. J Bacteriol 176, 6677-6687.

Doggett, R. G. (1969). Incidence of mucoid Pseudomonas aeruginosa from clinical sources. Appl Microbiol 18, 936-937.

Doggett, R. G., Harrison, G. M., Stillwell, R. N. \& Wallis, E. S. (1966). An atypical Pseudomonas aeruginosa associated with cystic fibrosis of the pancreas. J Pediatr 68, 215-221.

Drenkard, E. \& Ausubel, F. M. (2002). Pseudomonas biofilm formation and antibiotic resistance are linked to phenotypic variation. Nature 416, 740-743.

Evans, L. R. \& Linker, A. (1973). Production and characterization of the slime polysaccharide of Pseudomonas aeruginosa. J Bacteriol 116, 915-924.

Firoved, A. M., Boucher, J. C. \& Deretic, V. (2002). Global genomic analysis of $\mathrm{AlgU}\left(\sigma^{\mathrm{E}}\right)$-dependent promoters (sigmulon) in Pseudomonas aeruginosa and implications for inflammatory processes in cystic fibrosis. J Bacteriol 184, 1057-1064.

Fluge, G., Ojeniyi, B., Høiby, N., Digranes, A., Ciofu, O., Hunstad, E., Haanaes, O. C. \& Storrosten, O. T. (2001). Typing of Pseudomonas aeruginosa strains in Norwegian cystic fibrosis patients. Clin Microbiol Infect 7, 238-243.

Frederiksen, B., Koch, C. \& Høiby, N. (1997). Antibiotic treatment of initial colonization with Pseudomonas aeruginosa postpones chronic infection and prevents deterioration of pulmonary function in cystic fibrosis. Pediatr Pulmonol 23, 330-335.

Fyfe, J. A. \& Govan, J. R. (1980). Alginate synthesis in mucoid Pseudomonas aeruginosa: a chromosomal locus involved in control. J Gen Microbiol 119, 443-450.

Goldberg, J. B. \& Dahnke, T. (1992). Pseudomonas aeruginosa AlgB, which modulates the expression of alginate, is a member of the NtrC subclass of prokaryotic regulators. Mol Microbiol 6, 59-66.

Goldberg, J. B., Gorman, W. L., Flynn, J. L. \& Ohman, D. E. (1993). A mutation in algN permits trans activation of alginate production by algT in Pseudomonas species. J Bacteriol 175, 1303-1308.

Govan, J. R. \& Deretic, V. (1996). Microbial pathogenesis in cystic fibrosis: mucoid Pseudomonas aeruginosa and Burkholderia cepacia. Microbiol Rev 60, 539-574.
Govan, J. R., Fyfe, J. A. \& McMillan, C. (1979). The instability of mucoid Pseudomonas aeruginosa: fluctuation test and improved stability of the mucoid form in shaken culture. J Gen Microbiol 110, 229-232.

Govan, J. R., Fyfe, J. A. \& Baker, N. R. (1983). Heterogeneity and reduction in pulmonary clearance of mucoid Pseudomonas aeruginosa. Rev Infect Dis 5 (Suppl. 5), S874-S879.

Haussler, S. (2004). Biofilm formation by the small colony variant phenotype of Pseudomonas aeruginosa. Environ Microbiol 6, 546-551.

Hoffmann, N., Rasmussen, T. B., Jensen, P. O., Stub, C., Hentzer, M., Molin, S., Ciofu, O., Givskov, M., Johansen, H. K. \& Høiby, N. (2005). Novel mouse model of chronic Pseudomonas aeruginosa lung infection mimicking cystic fibrosis. Infect Immun 73, 2504-2514.

Høiby, N. (1975). Prevalence of mucoid strains of Pseudomonas aeruginosa in bacteriological specimens from patients with cystic fibrosis and patients with other diseases. Acta Pathol Microbiol Scand Suppl 83, 549-552.

Høiby, N. (1977). Pseudomonas aeruginosa infection in cystic fibrosis. Diagnostic and prognostic significance of Pseudomonas aeruginosa precipitins determined by means of crossed immunoelectrophoresis. A survey. Acta Pathol Microbiol Scand Suppl 262, 1-96.

Høiby, N., Giwercman, B., Jensen, E. T., Johansen, H. K., Kronborg, G., Pressler, T. \& Kharazmi, A. (1993). Immune response in cystic fibrosis helpful or harmful? In 18th European CF Conference, pp. 133-139. Edited by H. Escobar, F. Baquero \& L. Suarez. Madrid: Elsevier.

Høiby, N., Frederiksen, B. \& Pressler, T. (2005). Eradication of early Pseudomonas aeruginosa infection. J Cyst Fibros 4 (Suppl. 2), 49-54.

Jelsbak, L., Johansen, H. K., Frost, A. L., Thøgersen, R., Thomsen, L. E., Ciofu, O., Yang, L., Haagensen, J. A., Høiby, N. \& Molin, S. (2007). Molecular epidemiology and dynamics of Pseudomonas aeruginosa populations in lungs of cystic fibrosis patients. Infect Immun 75, 2214-2224.

Kessler, B., de Lorenzo, V. \& Timmis, K. N. (1992). A general system to integrate lac $Z$ fusions into the chromosomes of gram-negative eubacteria: regulation of the $\mathrm{Pm}$ promoter of the TOL plasmid studied with all controlling elements in monocopy. Mol Gen Genet 233, 293-301.

Knutson, C. A. \& Jeanes, A. (1968). A new modification of the carbazole analysis: application to heteropolysaccharides. Anal Biochem 24, 470-481.

Lee, B., Haagensen, J. A., Ciofu, O., Andersen, J. B., Høiby, N. \& Molin, S. (2005). Heterogeneity of biofilms formed by nonmucoid Pseudomonas aeruginosa isolates from patients with cystic fibrosis. J Clin Microbiol 43, 5247-5255.

Martin, D. W., Schurr, M. J., Mudd, M. H. \& Deretic, V. (1993a). Differentiation of Pseudomonas aeruginosa into the alginate-producing form: inactivation of $m u c B$ causes conversion to mucoidy. $\mathrm{Mol}$ Microbiol 9, 497-506.

Martin, D. W., Schurr, M. J., Mudd, M. H., Govan, J. R., Holloway, B. W. \& Deretic, V. (1993b). Mechanism of conversion to mucoidy in Pseudomonas aeruginosa infecting cystic fibrosis patients. Proc Natl Acad Sci U S A 90, 8377-8381.

Martin, D. W., Schurr, M. J., Yu, H. \& Deretic, V. (1994). Analysis of promoters controlled by the putative sigma factor AlgU regulating conversion to mucoidy in Pseudomonas aeruginosa: relationship to $\sigma^{\mathrm{E}}$ and stress response. J Bacteriol 176, 6688-6696.

Mathee, K., McPherson, C. J. \& Ohman, D. E. (1997). Posttranslational control of the $\operatorname{alg} T(\operatorname{alg} U)$-encoded $\sigma^{22}$ for expression of the alginate regulon in Pseudomonas aeruginosa and localization of its antagonist proteins MucA and MucB (AlgN). J Bacteriol 179, 3711-3720. 
Mathee, K., Ciofu, O., Sternberg, C., Lindum, P. W., Campbell, J. L., Jensen, P., Johnsen, A. H., Givskov, M., Ohman, D. E. \& other authors (1999). Mucoid conversion of Pseudomonas aeruginosa by hydrogen peroxide: a mechanism for virulence activation in the cystic fibrosis lung. Microbiology 145, 1349-1357.

Nikolskaya, A. N. \& Galperin, M. Y. (2002). A novel type of conserved DNA-binding domain in the transcriptional regulators of the AlgR/ AgrA/LytR family. Nucleic Acids Res 30, 2453-2459.

Ohman, D. E. (1981). Genetic mapping of chromosomal determinants for the production of exopolysaccharide alginate in a Pseudomonas aeruginosa cystic fibrosis isolate. Infect Immun 33, 142-148.

Ojeniyi, B., Høiby, N. \& Rosdahl, V. T. (1991). Genome fingerprinting as a typing method used on polyagglutinable Pseudomonas aeruginosa isolates from cystic fibrosis patients. APMIS 99, 492-498.

Ojeniyi, B., Petersen, U. S. \& Høiby, N. (1993). Comparison of genome fingerprinting with conventional typing methods used on Pseudomonas aeruginosa isolates from cystic fibrosis patients. APMIS 101, 168-175.

Palma, M., DeLuca, D., Worgall, S. \& Quadri, L. E. (2004). Transcriptome analysis of the response of Pseudomonas aeruginosa to hydrogen peroxide. J Bacteriol 186, 248-252.

Pedersen, S. S. (1992). Lung infection with alginate-producing, mucoid Pseudomonas aeruginosa in cystic fibrosis. APMIS Suppl $\mathbf{2 8}$, $1-79$.

Pedersen, S. S., Espersen, F., Høiby, N. \& Shand, G. H. (1989), Purification, characterization, and immunological cross-reactivity of alginates produced by mucoid Pseudomonas aeruginosa from patients with cystic fibrosis. J Clin Microbiol 27, 691-699.

Pugashetti, B. K., Metzger, H. M., Jr, Vadas, L. \& Feingold, D. S. (1982). Phenotypic differences among clinically isolated mucoid Pseudomonas aeruginosa strains. J Clin Microbiol 16, 686-691.

Römling, U. \& Tümmler, B. (2000). Achieving $100 \%$ typeability of Pseudomonas aeruginosa by pulsed-field gel electrophoresis. J Clin Microbiol 38, 464-465.

Rowen, D. W. \& Deretic, V. (2000). Membrane-to-cytosol redistribution of ECF sigma factor AlgU and conversion to mucoidy in Pseudomonas aeruginosa isolates from cystic fibrosis patients. Mol Microbiol 36, 314-327.

Schurr, M. J., Martin, D. W., Mudd, M. H. \& Deretic, V. (1994). Gene cluster controlling conversion to alginate-overproducing phenotype in Pseudomonas aeruginosa: functional analysis in a heterologous host and role in the instability of mucoidy. J Bacteriol 176, 3375-3382.

Schurr, M. J., Yu, H., Martinez-Salazar, J. M., Boucher, J. C. \& Deretic, V. (1996). Control of AlgU, a member of the $\sigma^{\mathrm{E}}$-like family of stress sigma factors, by the negative regulators MucA and MucB and Pseudomonas aeruginosa conversion to mucoidy in cystic fibrosis. J Bacteriol 178, 4997-5004.
Speert, D. P., Farmer, S. W., Campbell, M. E., Musser, J. M., Selander, R. K. \& Kuo, S. (1990). Conversion of Pseudomonas aeruginosa to the phenotype characteristic of strains from patients with cystic fibrosis. J Clin Microbiol 28, 188-194.

Tart, A. H., Blanks, M. J. \& Wozniak, D. J. (2006). The AlgT-dependent transcriptional regulator AmrZ (AlgZ) inhibits flagellum biosynthesis in mucoid, nonmotile Pseudomonas aeruginosa cystic fibrosis isolates. J Bacteriol 188, 6483-6489.

Tenover, F. C., Arbeit, R. D., Goering, R. V., Mickelsen, P. A., Murray, B. E., Persing, D. H. \& Swaminathan, B. (1995). Interpreting chromosomal DNA restriction patterns produced by pulsed-field gel electrophoresis: criteria for bacterial strain typing. J Clin Microbiol 33, 2233-2239.

Terry, J. M., Pina, S. E. \& Mattingly, S. J. (1991). Environmental conditions which influence mucoid conversion Pseudomonas aeruginosa PAO1. Infect Immun 59, 471-477.

Wood, L. F. \& Ohman, D. E. (2006). Independent regulation of MucD, an HtrA-like protease in Pseudomonas aeruginosa, and the role of its proteolytic motif in alginate gene regulation. J Bacteriol 188, 3134-3137.

Wood, L. F., Leech, A. J. \& Ohman, D. E. (2006). Cell wall-inhibitory antibiotics activate the alginate biosynthesis operon in Pseudomonas aeruginosa: roles of sigma (AlgT) and the AlgW and Prc proteases. Mol Microbiol 62, 412-426.

Wozniak, D. J. \& Ohman, D. E. (1991). Pseudomonas aeruginosa AlgB, a two-component response regulator of the $\mathrm{NtrC}$ family, is required for algD transcription. J Bacteriol 173, 1406-1413.

Wyckoff, T. J., Thomas, B., Hassett, D. J. \& Wozniak, D. J. (2002). Static growth of mucoid Pseudomonas aeruginosa selects for nonmucoid variants that have acquired flagellum-dependent motility. Microbiology 148, 3423-3430.

Yoon, S. S., Coakley, R., Lau, G. W., Lymar, S. V., Gaston, B., Karabulut, A. C., Hennigan, R. F., Hwang, S. H., Buettner, G. \& other authors (2006). Anaerobic killing of mucoid Pseudomonas aeruginosa by acidified nitrite derivatives under cystic fibrosis airway conditions. J Clin Invest 116, 436-446.

Yu, H., Schurr, M. J. \& Deretic, V. (1995). Functional equivalence of Escherichia coli sigma E and Pseudomonas aeruginosa AlgU: E. coli $r p o E$ restores mucoidy and reduces sensitivity to reactive oxygen intermediates in alg $U$ mutants of $P$. aeruginosa. J Bacteriol 177, 3259-3268.

Yu, H., Boucher, J. C., Hibler, N. S. \& Deretic, V. (1996). Virulence properties of Pseudomonas aeruginosa lacking the extreme-stress sigma factor AlgU (sigma E). Infect Immun 64, 2774-2781.

Edited by: P. Cornelis 